\title{
Entropy Production in the Expanding Universe ${ }^{\dagger}$
}

\author{
Mehrnoosh Farahmand * and Hosein Mohammadzadeh \\ Department of Physics, University of Mohaghegh Ardabili, P.O. Box 179, 56199-11367 Ardabil, Iran; \\ h.mohammadzadeh@gmail.com \\ * Correspondence: mehrnoosh.farahmandrad@gmail.com \\ + Presented at the 4th International Electronic Conference on Entropy and Its Applications, Basel, Switzerland, \\ 21 November-1 December 2017. Available online: http:/ / sciforum.net/conference/ecea-4.
}

Published: 23 November 2017

\begin{abstract}
The spacetime is basically curved and dynamical. Thus our knowledge of universe must be extended to a dynamical curved spacetime to understand the nature of the universe. The field theory in the curved spacetime has shown that the evolution of spacetime involving the field in the curved spacetime leads to particle creation. From another perspective, by employing thermodynamics to cosmology, we can learn about the source of current entropy content associated with the universe. From the quantum thermodynamics, it is clear that the inner friction stemming from the quantum fluctuations of the field can produce the entropy. Using this approach, the particle creation due to the expansion of spacetime beginning from the vacuum is shown as an entropic increase. Considering an asymptotically flat Robertson-Walker spacetime, the particle creation entropy is evaluated. Each special scale factor can be used to characterize the cosmic parameters. Thus, the dependence of particle creation entropy on the field parameters and the cosmic parameters allows us to recover information from the underlying structure of the spacetime. Also, by adding an entropy production, indicating the mutual information between created particle and spacetime, to this particle creation entropy, the well-known entanglement measure can obtained to investigate the entanglement of created particles. In fact, the entanglement entropy, measuring the mixedness of the primary state, is affected from the creation and the correlation of the particle.
\end{abstract}

Keywords: quantum thermodynamics entropy; quantum entanglement entropy; mutual information entropy; expanding universe

PACS: 04.62.+v; 03.65.-w; 03.65.Ud; 03.65.Yz

\section{Introduction}

The dynamical behavior of curved spacetime is considered to discover the phenomenon of the universe. The dynamics of fields living in spacetime is affected by the structure of spacetime. The quantum field theory in curved spacetime leads to particle creation, stemming from the interaction between the quantum field and gravitational field [1].

The entanglement of created particles in curved spacetime and its applications such as quantum information protocols in the relativistic limit is well-known for understanding of the nature of the universe [2-17]. The von Neumann entropy of created particles as a measure of entanglement is often applied to discover of the encoded information of spacetime [2,3]. From another direction, the entanglement has been employed in identifying the thermodynamic properties of the spacetime structure [18].

Thermodynamics is applied to describe small-scale and large-scale systems, such as black holes, dark matter and cosmology [19-21]. The study of the thermodynamic properties of quantum fields in the expanding universes has shown that an inner friction due to quantum fluctuations of fields can be interpreted as an entropic increase, given by quantum fluctuations [22,23]. 
We start with an asymptotically spatially flat Robertson-Walker spacetime using an special scale factor [24], which is the generalization of various particular cases found in literature and evaluate the particle creation entropy in the distant future from the distant past vacuum. We show that one can explore particle creation entropy as a tool for characterising the cosmological parameters of each Robertson Walker universe.

An exact expression has been obtained for deriving the entropy production of a system that is in contact with a thermal reservoir with strong coupling between system and reservoirs [25]. The system entropy production has been recognised as a direct measure of the system-reservoir correlations and/or entanglements. The entropy production has been established as a measure for the information lost in the correlations between the system and the reservoir via the mutual information [26]. Our framework is described such that the field and the underlying spacetime are two interacting systems, if the spacetime is initially in a thermal state and plays the role of the reservoir that drives the field towards equilibrium. We will explore the relation between the particle creation entropy, the von Neumann entropy and the entropy production. Therefore, the mutual information as entropy production will be obtained as a measure for the correlation between created particle and spacetime.

\section{Particle Creation in the Expanding Universe}

A $1+3$ dimensions expanding Robertson-Walker spacetime is provided with a metric of the form

$$
d s^{2}=\Omega^{2}(\eta)\left(d \eta^{2}-\sum_{i=1}^{3}\left(d x^{i}\right)^{2}\right)
$$

where $\Omega$ is the conformal scale factor and $\eta$ is the conformal time parameter, $d \eta=a^{-1} d t$. We need that the metric, Equation (1), is conformally flat, with a time-like conformal Killing vector. For such a metric the conformal factor $\Omega(\eta)$ is constant as $\eta \rightarrow \pm \infty$. Thus the observers flowing along the asymptotically time-like Killing vector can describe particle states [1].

A massive scalar field, as inflationary scenario [27], leads to the positive and negative frequency solutions for the Klein-Gordon equation in the spacetime of Equation (1). Therefore, the equation of motion of the scalar field is described by

$$
\left[+m^{2}\right] \phi=0
$$

where the d'Alambertian operator in a curved spacetime is $\phi:=g^{\mu \nu} \nabla_{\mu} \nabla_{\nu} \phi=\frac{1}{\sqrt{-g}} \partial_{\mu}\left(\sqrt{-g} g^{\mu \nu} \partial_{\nu} \phi\right)$. For the quantization of the field, we decompose it in terms of the positive and negative mode solutions of the Klein-Gordon equation, $u_{k}(\eta, x)$, as follows

$$
\phi(\eta, x)=\int d^{3} k\left[a_{k} u_{k}(\eta, x)+a_{k}^{\dagger} u_{k}^{*}(\eta, x)\right],
$$

where $k=|\mathbf{k}|, a_{k}, a_{k}^{\dagger}$ are annihilation and creation operators, respectively, and $\left(u_{k}, u_{k^{\prime}}\right)=\delta_{k k^{\prime}}$, $\left(u_{k^{\prime}}^{*}, u_{k^{\prime}}^{*}\right)=-\delta_{k k^{\prime}},\left(u_{k}, u_{k^{\prime}}^{*}\right)=0$. The invariance with respect to the spatial translation requires that the solutions are separable into spatial and time components as follows

$$
u_{k}=(2 \pi)^{-\frac{3}{2}} e^{i k \cdot x} \chi_{k}(\eta)
$$

Thus we obtain the simplified equation of motion as follows

$$
\frac{d^{2} \chi_{k}}{d \eta^{2}}+\omega^{2}(\eta) \chi_{k}=0
$$

where 


$$
\omega^{2}=k^{2}+m^{2} \Omega^{2}(\eta)
$$

In the following, two sets of the solutions $\chi_{\text {in }}^{ \pm}$and $\chi_{\text {out }}^{ \pm}$represent the behavior of positive and negative frequency solutions at $\eta \rightarrow-\infty$ and $\eta \rightarrow+\infty$, respectively. By choosing the following form for the scale factor

$$
\Omega^{2}=a+b \tanh (\lambda \eta)+c \tanh ^{2}(\lambda \eta)
$$

and changing $\eta \rightarrow \tau$ such as follows

$$
\tau=\frac{1+\tanh (\lambda \eta)}{2}
$$

The solving of the resulting equation gives rise to two sets of solutions in terms of hypergeometric functions as follows

$$
\chi_{\text {in }}^{ \pm}(\tau)=\frac{1}{\sqrt{2 \omega_{\text {in }}}} \tau^{\mp i \frac{\omega_{\text {in }}}{2 \lambda}}(1-\tau)^{ \pm i \frac{\omega_{\text {out }}}{2 \lambda}} F\left(\frac{1}{2} \pm i \frac{\omega_{-}}{\lambda}+i \delta, \frac{1}{2} \pm i \frac{\omega_{-}}{\lambda}-i \delta ; 1 \mp i \frac{\omega_{\text {in }}}{\lambda} ; \tau\right),
$$

and

$$
\chi_{\text {out }}^{ \pm}(\tau)=\frac{1}{\sqrt{2 \omega_{\text {out }}}} \tau^{\mp i \frac{\omega_{\text {in }}}{2 \lambda}}(1-\tau)^{ \pm i \frac{\omega_{\text {out }}}{2 \lambda}} F\left(\frac{1}{2} \pm i \frac{\omega_{-}}{\lambda}+i \delta, \frac{1}{2} \pm i \frac{\omega_{-}}{\lambda}-i \delta ; 1 \pm i \frac{\omega_{\text {out }}}{\lambda} ; 1-\tau\right),
$$

with

$$
\begin{gathered}
\omega_{\text {in }}=\sqrt{k^{2}+m^{2}(a+c-b)}, \\
\omega_{\text {out }}=\sqrt{k^{2}+m^{2}(a+c+b)}, \\
\omega_{ \pm}=\frac{\omega_{\text {out }} \pm \omega_{\text {in }}}{2},
\end{gathered}
$$

and

$$
\delta=\frac{1}{2} \sqrt{\frac{4 m^{2} c}{\lambda^{2}}-1} .
$$

The vacuum state of the field in the past infinity is considered as a thermal state from the perspective of an observer in the distant future. So by using the properties of hypergeometric functions, we can benefit from the Bogoliubov transformation to connect the "in" and "out" solutions.

$$
\begin{aligned}
& \chi_{\text {in }}^{+}=\alpha \chi_{\text {out }}^{+}+\beta \chi_{\text {out }}^{-} \\
& \chi_{\text {in }}^{+}=\beta^{*} \chi_{\text {out }}^{+}+\alpha^{*} \chi_{\text {out }}^{-} .
\end{aligned}
$$

Therefore, the Bogoliubov coefficients $\alpha$ and $\beta$ are obtained by

$$
\alpha=\sqrt{\frac{\omega_{\mathrm{out}}}{\omega_{\text {in }}}} \frac{\Gamma\left(1-i \frac{\omega_{\text {in }}}{\lambda}\right) \Gamma\left(-i \frac{\omega_{\text {out }}}{\lambda}\right)}{\Gamma\left(\frac{1}{2}-i \frac{\omega_{+}}{\lambda}-i \frac{1}{2} \sqrt{\frac{4 m^{2} c}{\lambda}-1}\right) \Gamma\left(\frac{1}{2}-i \frac{\omega_{+}}{\lambda}+i \frac{1}{2} \sqrt{\frac{4 m^{2} c}{\lambda}-1}\right)},
$$

and

$$
\beta=\sqrt{\frac{\omega_{\text {out }}}{\omega_{\text {in }}}} \frac{\Gamma\left(1-i \frac{\omega_{\text {in }}}{\lambda}\right) \Gamma\left(i \frac{\omega_{\text {out }}}{\lambda}\right)}{\Gamma\left(\frac{1}{2}+i \frac{\omega_{-}}{\lambda}-i \frac{1}{2} \sqrt{\frac{4 m^{2} c}{\lambda}-1}\right) \Gamma\left(\frac{1}{2}+i \frac{\omega_{-}}{\lambda}+i \frac{1}{2} \sqrt{\frac{4 m^{2} c}{\lambda}-1}\right)} .
$$

Therefore, the creation an annihilation operators in far future corresponds to a linear combination of these operators in far past, relating to each other by Bogoliubov coefficients as follows

$$
\begin{aligned}
& a_{\text {out }}=\alpha a_{\text {in }}+\beta^{*} b_{\text {in }}^{+} \\
& b_{\text {out }}^{+}=\beta a_{\text {in }}+\alpha^{*} b_{\text {in }}^{+}
\end{aligned}
$$


where from canonical commutation relations for the above operators, we have

$$
|\alpha|^{2}+|\beta|^{2}=1
$$

We define $\left|0_{\text {in }}\right\rangle$ as the initial vacuum state at $\eta \rightarrow+\infty$, in which there are no particles. Therefore, for the exited state as $\eta \rightarrow+\infty$, the number of particles with respect to the $k$ th mode is given by

$$
\left\langle N_{k}\right\rangle=\left\langle 0_{\text {in }}\left|\tilde{a}_{\text {out }}^{\dagger} \tilde{a}_{\text {out }}\right| 0_{\text {in }}\right\rangle .
$$

Therefore, we can find

$$
n_{\mathrm{cr}}=|\beta|^{2},
$$

where we have denoted $\left\langle N_{k}\right\rangle \equiv n_{\mathrm{cr}}$. As the conclusion, the "in" vacuum (far past) regime is in corresponding to the excited states in "out" vacuum regime. Using Equation (17) the number of particle creation gives

$$
n_{\mathrm{cr}}=\frac{\cosh \left(2 \pi \frac{\omega_{-}}{\lambda}\right)+\cosh \left(\pi \sqrt{\frac{4 m^{2} c}{\lambda^{2}}-1}\right)}{\cosh \left(2 \pi \frac{\omega_{+}}{\lambda}\right)-\cosh \left(2 \pi \frac{\omega_{-}}{\lambda}\right)} .
$$

\section{Particle Creation Entropy}

In the beginning of the spacetime, the universe undergoes a rapid expansion. Therefore, in this section we concentrate on two modes of opposite momentum $k$ and $-k$. The interaction between this field and any other external fields is ignorable and the mode pair of the field is correlated through two-mode squeezing [28-30]. Thus our mode pair undergoes a unitary evolution. We choose the thermodynamical framework such that the pair of field modes is regarded as a system and the spacetime is regarded as an energy source which can change the inertial energy of the field. In this way, the mode pairs and external spacetime constitute a closed system, as spacetime is expanding. The expansion of the spacetime is doing work on the quantum field. The asymptotic past and future Hamiltonians are found by

$$
\begin{aligned}
& H_{\text {in }}=\omega_{\text {in }}\left(a_{\text {in }}^{+} a_{\text {in }}+b_{\text {in }}^{\dagger} b_{\text {in }}+1\right), \\
& H_{\text {out }}=\omega_{\text {out }}\left(a_{\text {out }}^{+} a_{\text {out }}+b_{\text {out }}^{+} b_{\text {out }}+1\right),
\end{aligned}
$$

respectively. When the spacetime changes, work is being done on the quantum field, and an energy variation induces in the modes of opposite momentum of the field. This unitary evolution takes the field away from equilibrium. The average work is obtained by

$$
W \equiv \operatorname{Tr}\left[H_{\text {out }} \rho\right]-\operatorname{Tr}\left[H_{\text {in }} \rho\right]=\omega_{\text {out }} n_{\text {cr }}+\left(\omega_{\text {out }}-\omega_{\text {in }}\right),
$$

where $\rho$ is the state of the mode pair. The Equation (24) is the sum of the energy of created particles and the change in ground state energy, in which the latter is related to the quantum adiabatic expansion of the universe. The difference between the average work and the quantum adiabatic work is known as friction work [31-33].

$$
W_{\text {friction }}=W-W_{\text {adiabatic }}=\omega_{\text {out }} n_{\mathrm{cr}} .
$$

From the fluctuation theorem [23,34-36], it has been shown that the inner friction $\langle W\rangle_{\text {friction }}$ gives rise to the entropy production during the particle creation, stemming from evolution of spacetime [22]. The entropy production, $s$, is found as

$$
s=\frac{W_{\text {friction }}}{T}=\frac{\omega_{\text {out }}}{T} n_{\mathrm{cr}} .
$$


In order to find temperature $T$, we note that the pure vacuum separable state in the far past will be an entangled state in the far future. Therefore, the vacuum state in "in" region as a two-mode squeezed state can be written as a Schmidt decomposition of "out" state as follows

$$
\left|0_{\text {in }}\right\rangle=\left|0_{k} 0_{-k}\right\rangle=\sum_{n} A_{n}\left|n_{k}\right\rangle\left|n_{-k}\right\rangle .
$$

The definition of the vacuum state, $a_{\text {in }}\left|0_{\text {in }}\right\rangle=0$, and Equation (18) imply the relation $A_{n}=\left(\frac{\beta^{*}}{\alpha^{*}}\right)^{n} A_{0}$. Using the normalization condition $\left\langle 0_{\text {in }} \mid 0_{\text {in }}\right\rangle=1$, the vacuum state in the far past is as follows

$$
\left|0_{\text {in }}\right\rangle=\frac{1}{|\alpha|} \sum_{n=0}^{\infty}\left(\frac{\beta^{*}}{\alpha^{*}}\right)^{n}\left|n_{k}\right\rangle\left|n_{-k}\right\rangle
$$

The reduced density matrix is achieved by tracing out one of the two modes.

$$
\rho=\operatorname{Tr}_{-k}\left[\left|0_{\text {in }}\right\rangle\left\langle 0_{\text {in }}\right|\right]
$$

Then we obtain

$$
\rho=\frac{1}{|\alpha|^{2}} \sum_{n}\left(\frac{|\beta|}{|\alpha|}\right)^{2 n}\left|n_{k}\right\rangle\left\langle n_{k}\right|
$$

A simple calculation shows that the Equation (30) is a thermalized state.

$$
\rho=\left(1-e^{-\frac{\omega_{\text {out }}}{T}}\right) \sum_{n_{k}} e^{-\frac{\omega_{\text {out }}}{T} n_{k}}\left|n_{k}\right\rangle\left\langle n_{k}\right|,
$$

where we define the Bogoliubov coefficients from Equation (19) as $|\alpha| \equiv \cosh z$ and $|\beta| \equiv \sinh z, z$ is the squeezing parameter [37], $\tanh z \equiv e^{-\frac{\omega_{\text {out }}}{T}}$ and $\frac{\omega_{\text {out }}}{2}$ is related to the ground state energy of the positive modes in Equation (28). Therefore, the density number of the particle creation is a thermal Bose-Einstein distribution,

$$
n_{\mathrm{cr}}=\frac{1}{e^{\frac{\omega_{\text {out }}}{T}}-1}=|\beta|^{2}
$$

Now, we can extract the temperature from Equation (32) as follows

$$
T=\frac{\omega_{\text {out }}}{\log \left(\frac{1}{\gamma}\right)}
$$

where

$$
\gamma \equiv\left|\frac{\alpha}{\beta}\right|^{2}
$$

The Equation (33) is known as Unruh temperature [38]. Hence, the entropy of particle creation is found by using Equations (21), (26) and (33),

$$
S_{\mathrm{cr}}=s=\log \left(\frac{1+n_{\mathrm{cr}}}{n_{\mathrm{cr}}}\right)^{n_{\mathrm{cr}}} .
$$

\section{Interpretation of Results}

let us in this section discuss the obtained results. We notice that the form of scale factor Equation (7) is a generalisation of some particular cosmological models, found in literature [39-41]. With particular choices of parameters $a, b$ and $c$ we get some well-known models such as radiation dominated universe, which is a case with $a=b=0, c=\frac{a_{0}^{4}}{4 \lambda^{2}}$ and taking the limit $\lambda \rightarrow 0$ or with $c=0$, we have the 
cosmological model $\Omega^{2}(\eta)=a+b \tanh (\lambda \eta)$, widely studied in $[39,40]$ and also a particular choice of parameters $a=\left(\frac{1+\epsilon}{2}\right)^{2}, b=\frac{1-\epsilon^{2}}{2}$ and $c=\left(\frac{1-\epsilon}{2}\right)^{2}$ gives rise to the model discussed in [41].

Figures 1 and 2 represent particle creation entropy in terms of the field parameters, i.e., the mass and momentum of any modes, in based on the two latter models. The entropy of massive scalar modes turns out to be a monotonic decreasing function of the momentum modes such that the zero momentum modes have the maximum value of entropy. Also, this entropy becomes maximum with respect to the mass of the modes at a same certain mass, $m_{\max }$, for each $k$ mode. In conclusion, we have obtained the same results as those found in $[2-4,9]$. Thus with following the approach in $[3,9]$ to evaluate of the cosmological parameters, i.e., $a, b$ and $c$, we can get some information from the structure of the spacetime in which the field lives. The radiation dominated universe has been plotted in Figure 3. As is observed, for the radiation dominated universe the behavior of $S_{\mathrm{cr}}$ is different with respect to the other models. For the radiation dominated universe spectral behavior of the particle creation entropy shows that the entropy with respect to momentum modes has a maximum. That means there is a specific momentum for each mode, $k_{\max }$, at which the entropy is maximum. In fact, entropy peaks at a certain momentum. This means that modes of this characteristic frequency are far more prone to creation and correlation than any others. For the larger momentum modes, the entropy reaches to a constant value. In fact, for a large enough entropy all momentum modes are present. Since the creation of superheavy particles in the early universe has important cosmological consequences [42-45], we can conclude also that creation of superheavy particles may be important in this case. On the other hand, in general when $m \gg 1$, then $S_{\mathrm{cr}} \rightarrow 0$. However, when $a-b+c=0$ or $c>a+b$ then $S_{\mathrm{cr}} \rightarrow$ const $>0$ even if $m \gg 1$. This implies that it is possible to create superheavy particles in this model. In Figures 4 and 5a similar pattern of behavior to that of radiation dominated case, is observed for the particle creation entropy.

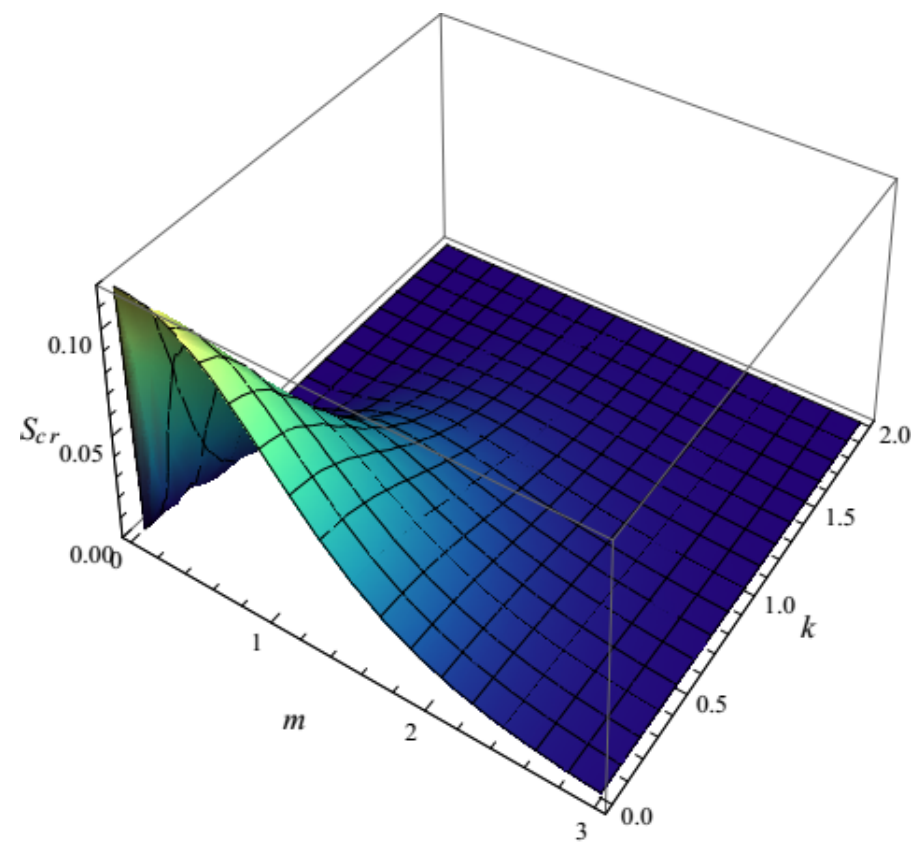

Figure 1. Particle creation entropy, $S_{\mathrm{cr}}$, as a function of momentum $k$ and mass $m$ for fixed values $a=1.5, b=1, c=0$ and $\lambda=2$. 


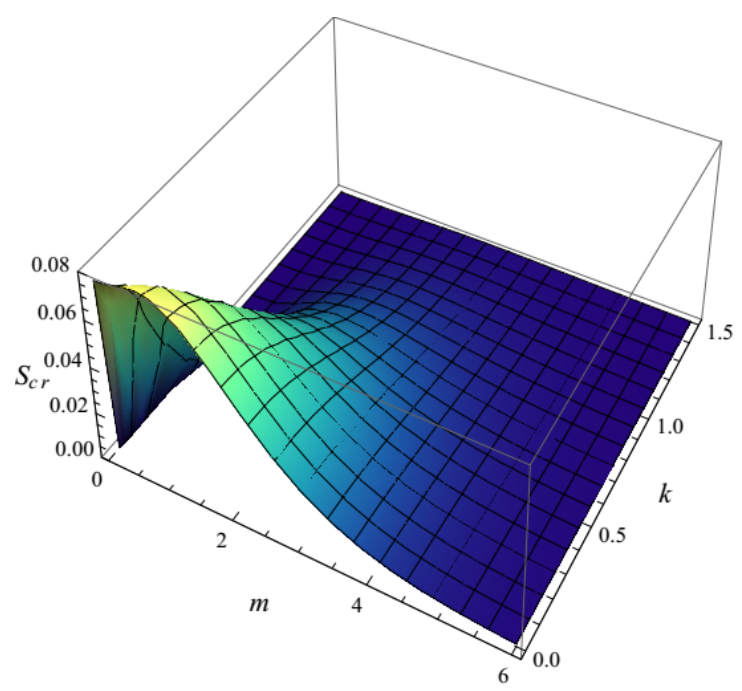

Figure 2. Particle creation entropy, $S_{\mathrm{cr}}$, as a function of momentum $k$ and mass $m$ for fixed values $a=\left(\frac{1+\epsilon}{2}\right)^{2}, b=\frac{1-\epsilon^{2}}{2}, c=\left(\frac{1-\epsilon}{2}\right)^{2}$ and $\lambda=1$.

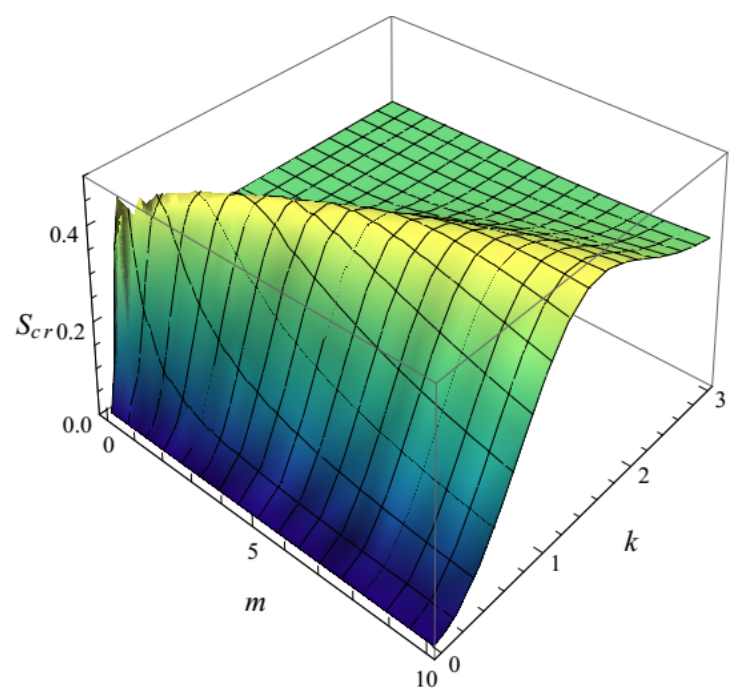

Figure 3. Particle creation entropy, $S_{\mathrm{cr}}$, as a function of momentum $k$ and mass $m$ for radiation dominated universe.

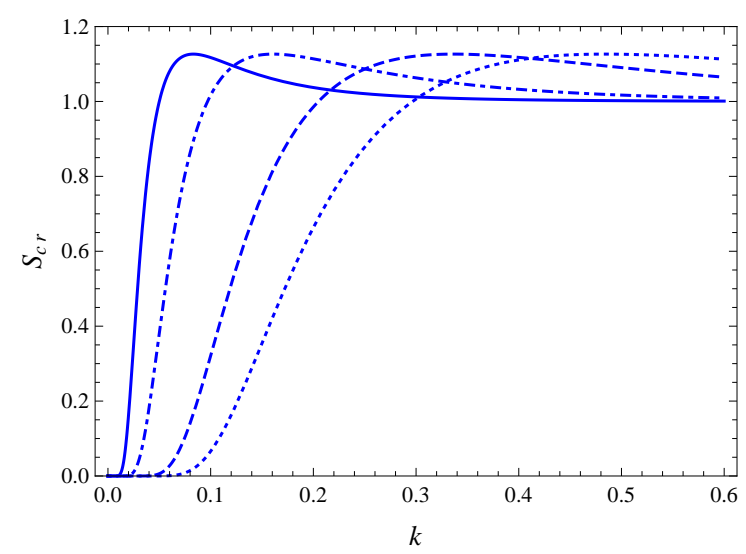

Figure 4. Particle creation entropy, $S_{\mathrm{cr}}$, as a function of momentum $k$ for fixed values $a=1, b=2, c=1$, $\lambda=1.5$ and the different values of mass $m=0.1$ (solid), $m=0.2$ (dotdashed), $m=0.5$ (dashed), and $m=1$ (dotted) curves. 


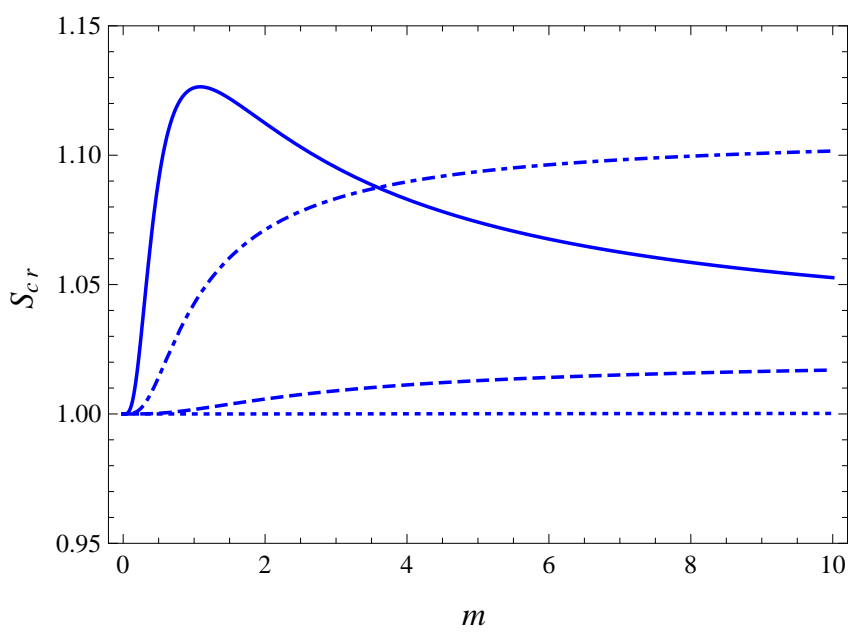

Figure 5. Particle creation entropy, $S_{\mathrm{cr}}$, as a function of mass $m$ for fixed values $a=1, b=2, c=1$, $\lambda=1.5$ and the different values of momentum $k=0.5$ (solid), $k=1$ (dotdashed), $k=2$ (dashed), and $k=4$ (dotted) curves.

\section{Entanglement Entropy}

For a pure separable state, which is initially the vacuum state in distant past, the new vacuum state is viewed as an entangled state from the perspective of a distant future observer. The entanglement measure of two systems, forming a pure state, has been defined by the von Neumann entropy of either of the reduced density matrices as follows [46].

$$
S(\rho)=-\operatorname{Tr}[\rho \log (\rho)]
$$

The eigenvalues of the reduced density matrix Equation (30) are $(1-\gamma) \gamma^{n}$. Using Equation (36) the von Neumann entropy, entanglement entropy, is calculated as follows

$$
S_{\mathrm{en}}=S(\rho)=\log \left(\frac{\gamma^{\frac{\gamma}{\gamma-1}}}{1-\gamma}\right) .
$$

The entanglement entropy can be rewritten by using Equation (21) as follows

$$
S_{\mathrm{en}}=\log \left(\frac{\left(1+n_{\mathrm{cr}}\right)^{n_{\mathrm{cr}}+1}}{n_{\mathrm{cr}} n_{\mathrm{cr}}}\right)
$$

The von Neumann entropy for a pure separable state is zero and the state function of such a state can completely describe it. In fact, repeating copies of a prepared pure separable state does not provide any new information about it. From the view point of a distant future observer, the primary system will become necessarily an entangled state because of the quantum fluctuations of the vacuum. In regard to entanglement, analysis of the system is performed through the von-Neumann entropy. Therefore, the initially separable state can be interpreted as a completely certain state, corresponding to zero entropy, while the dynamical spacetime, depending to the selected metric, will produce an entangled state with nonzero entropy. Indeed, if a state is seen as part of a pure entangled state, then the von Neumann entropy measures the entanglement in the total state.

\section{Entropy Production}

In this section, we extend the traditional framework of thermodynamics from an alternative viewpoint. We consider two interacting systems, one of the systems is initially in a thermal state and plays the role of the reservoir while the other is the driven system of interest. The initial state of the 
compound system does not display any entanglement or correlation. For such a framework, it has been shown that the system entropy production is always positive and is a direct measure of the system-reservoir correlations. In this set-up, it has been assumed that the total von Neumann entropy induces a natural separation of the entropy change of the system into separate contributions from an entropy flow and an entropy production and further erroneously suppose that the total entropy production is simply the sum of the system and reservoir entropy. Therefore, the change in the entropy of the system, which is a positive quantity, can be written in the standard thermodynamic form $[25,47]$

$$
S_{\mathrm{en}}=S_{\mathrm{pr}}+S_{\mathrm{cr}}
$$

where we can rewrite the entropy production as

$$
S_{\mathrm{pr}}=S_{\mathrm{en}}-S_{\mathrm{cr}}
$$

where $S_{\mathrm{en}}$ is the exact entropy change of the system, characterised by von Neumann entropy. The last term in (40) identifies the entropy flow, which can seen as the irreversible contribution to the system entropy change due to flow of energy from the reservoir to the system. The positive entropy production introduced above represents the irreversible contribution to the entropy change of the system, which indeed vanishes only when the system and the reservoir are totally decorrelated. In other words, the entropy production explicitly expresses how far the actual state of the total system is from the decorrelated product state [25].

In sec. III, the spacetime was supposed to play the role of a thermal reservoir such that together the mode pairs constitute a closed system as spacetime is expanding. This situation is similar to the second law of phenomenological non-equilibrium thermodynamics [47] and therefore can be modeled with Equation (40). We note that this model provides interesting insight into the notion of the entropy production. In based on, the initial total system state is decorrelated. The non-negative entropy production, characterising the irreversibility of a process, is given by the sum of the entropy change in the mode pairs and in the (macroscopic ideal and always equilibrated) spacetime. While there is no access to the state of the spacetime as the reservoir, which can be know solely from the energy that can exchange during its interaction with the mode pairs, the information lost in not knowing the state of the spacetime after the interaction can be negligible [26]. It relies on the assumption that we defined the spacetime as an ideal heat reservoir that remains close to thermal equilibrium during its interaction with the system. Indeed, this means that the entropy production due to an ideal heat reservoir coincides with the lost mutual information between the system and the reservoir [26]. Then the entropy production measures the information lost in the correlations between the system and the reservoir via the mutual information. In the other words, the entropy production explicitly expresses how far the actual state of the total system is from the decorrelated product state.

Correspondingly, if we consider the entropy balance by defining the von Neumann entropy of the system, interpreted as a measure for our lack of knowledge about the state of the system, also introduce particle creation entropy as the entropy flow, i.e., the system entropy change due to flow of energy from the spacetime to the field as particle creation, the non-negative (quantum) mutual information can be defined as [4]

$$
I=S_{\mathrm{pr}}=\log \left(1+n_{\mathrm{cr}}\right),
$$

which measures the amount of correlations shared between the created particle and the spacetime [48], vanishing only when the created particle and the spacetime are totally decorrelated. Consequently, the mutual information is a measure of the information lost when disregarding the correlation established between the created particle and the spacetime while keeping full knowledge of them separate in the description. 


\section{Conclusions}

We reviewed particle creation from vacuum in an asymptotically flat Robertson-Walker spacetime. Applying a scalar field in the spacetime leads to two sets of exact solutions for Klein-Gordon equation, based on a Bogoliubov transformation. We benefited from a special scale factor, which has been introduced in [24], and expressed the density of created particles.

The mode pairs $k$ and $-k$, which have unitary interaction during expansion, was modeled by two-mode squeezing. The two-mode squeezing system describes a thermal state, so that the mode pairs are in thermal equilibrium with respect to each other in the classical spacetime background. Therefore, one can find the equilibrium temperature, depending on the scale factor. Considering the mode pairs and underlying spacetime as a reservoir, forming a closed quantum state driven out of equilibrium by unitary evolution, the particles will be created and there will be an increase of entropy. We followed the formalism of evaluating particle creation entropy presented in [22]. The particle creation entropy, as understood in [36], is considered as the difference between the probability distributions obtained in the forward and the reverse process of expanding of the spacetime. In this way, the entropy production is proportional to inner friction work, which by creating the thermodynamic fluctuations, increases the entropy. The particle creation entropy was evaluated in terms of the specific scale factors considered for the spacetime [4]. It is worth mentioning that a scalar field in a dark energy dominated universe would also be interesting [49]. Then we have discussed some particular cosmological models, which is deduced via some choices for the cosmological parameter of particular scale factor. Correspondingly, investigation of the created entanglement was performed by applying the von Neumann entropy. Afterward, we derived the mutual information due to the correlation of the mode pairs and the spacetime. Our results show that the von Neumann entropy, evaluating the entanglement of the system, is the total of the created particles correlations and/or entanglement as the particle creation entropy and the mutual information due to the particle-spacetime correlations and/or entanglement as the production entropy. The comparison was shown that all entropy quantities can be treated in the same way, on equal footing, and all of them can be linked with each other and expressed as the increasing entropy of the universe due to particle creation [4]. Thus, using these quantities and dependence of them on the field parameters and cosmic parameters, it is possible to recover information from the underlying structure of the spacetime as one has been discussed in [5]. Therefore, the formulation of thermodynamics is generalized to quantum formalism. Entanglement can be considered as a source of thermodynamic properties related to the universe and employed in the general thermodynamic picture of the universe. In fact, entanglement presents an important tool to study observable consequences in cosmology.

Conflicts of Interest: The authors declare no conflict of interest.

\section{References}

1. Birrell, N.D.; Davies, P.C.W. Quantum Fields in Curved Space; Cambridge University Press: Cambridge, UK, 1982.

2. Ball, J.L.; Fuentes-Schuller, I.; Schuller, F.P. Entanglement in an expanding spacetime. Phys. Lett. A 2006, 359, 550-554.

3. Fuentes, I.; Mann, R.B.; Martín-Martínez, E.; Moradi, S. Entanglement of Dirac fields in an expanding spacetime. Phys. Rev. D 2010, 82, 045030.

4. Farahmand, M.; Mohammadzadeh, H.; Mehri-Dehnavi, H. Quantum thermodynamics and quantum entanglement entropies in an expanding universe. Int. J. Mod. Phys. 2017, 32, 1750066.

5. Mohammadzadeh, H.; Farahmand, M.; Maleki, M. Entropy production due to Lorentz invariance violation. Phys. Rev. D 2017, 96, 024001.

6. Martín-Martínez, E.;Menicucci, N.C. Entanglement in curved spacetimes and cosmology. Class. Quantum Gravity 2014, 31, 214001.

7. Ebadi, Z.; Mirza, B. Entanglement generation by electric field background. Ann. Phys. 2014, 351, 363-381. 
8. Ebadi, Z.; Mirza, B. Entanglement generation due to the background electric field and curvature of space-time. Int. J. Mod. Phys. A 2015, 30, 1550031.

9. Mohammadzadeh, H.; Ebadi, Z.; Mehri-Dehnavi, H.; Mirza, B.; Rahimi, R. Entanglement of arbitrary spin modes in expanding universe. Quantum Inf. Process. 2015, 14, 4787-4801.

10. Pierini, R.; Moradi, S.; Mancini, S. The role of spin in entanglement generated by expanding spacetime. Int. J. Theor. Phys. 2016, 55, 3059-3078.

11. Mann, R.B.; Ralph, T.C. Relativistic quantum information. Class. Quantum Gravity 2012, $29,220301$.

12. Benincasa, D.M.T.; Borsten, L.; Buck, M.; Dowker, F. Quantum information processing and relativistic quantum fields. Class. Quantum Gravity 2012, 31, 075007.

13. Alsing, P.M.; Milburn, G.J. Teleportation with a uniformly accelerated partner. Phys. Rev. Lett. 2003, 91, 180404.

14. Alsing, P.M.; Fuentes-Schuller, I.; Mann, R.B.; Tessier, T.E. Entanglement of Dirac fields in noninertial frames. Phys. Rev. A 2006, 74, 032326.

15. Adesso, G.; Fuentes-Schuller, I.; Ericsson, M. Continuous-variable entanglement sharing in noninertial frames. Phys. Rev. A 2007, 76, 062112.

16. Mehri-Dehnavi, H.; Rahimi, R.; Mohammadzadeh, H.; Ebadi, Z.; Mirza, B. Quantum teleportation with nonclassical correlated states in noninertial frames. Quantum Inf. Process. 2015, 14, 1025-1034.

17. Helder, A.; de Souza, G.; Mansfield, P.; Sampaio, M. Alice and Bob in an expanding spacetime. EPL 2015, 111,60001

18. Müller, R.; Lousto, C.O. Entanglement entropy in curved spacetimes with event horizons. Phys. Rev. D 1995, 52, 4512-4517.

19. Zare, S.; Raissi, Z.; Mohammadzadeh, H.; Mirza, B. Condensation of an ideal gas with intermediate statistics on the horizon. Eur. Phys. J. C 2012, 72, 2152.

20. Ebadi, Z.; Mirza, B.; Mohammadzadeh, H. Infinite statistics condensate as a model of dark matter. J. Cosmol. Astropart. Phys. 2013, 11, 057.

21. Parker, L. Particle creation in expanding universes. Phys. Rev. Lett. 1968, 21, 562.

22. Liu, N.; Goold, J.; Fuentes, I.; Vedral, V.; Modi, K.; Bruschi, D.E. Quantum thermodynamics for a model of an expanding universe. Class. Quantum Gravity 2016, 33, 035003.

23. Campisi, M.; Hänggi, P.; Talkner, P. Colloquium: Quantum fluctuation relations: Foundations and applications. Rev. Mod. Phys. 2011, 83, 771-791.

24. Haouat, S.; Chekireb, R. On the Creation of Scalar Particles in a Flat Robertson-Walker Spacetime. Mod. Phys. Lett. A 2011, 26, 2639-2651.

25. Esposito, M.; Lindenberg, K.; van den Broeck, C. Entropy production as correlation between system and reservoir. New J. Phys. 2010, 12, 013013.

26. Strasberg, P.; Schaller, G.; Brandes, T. Quantum and information thermodynamics: A unifying framework based on repeated interactions. Phy. Rev. X 2017, 7, 021003.

27. Mukhanov, V. Physical Foundations of Cosmology; Cambridge University Press: Cambridge, UK, 2005.

28. Alsing, P.M.; McMahon, D.H.; Milburn, G.J. Teleportation in a non-inertial frame. J. Opt. B Quantum Semiclass. Opt. 2004, 6, S834.

29. Yurke, B.; Potasek, M. Obtainment of thermal noise from a pure quantum state. Phys. Rev. A 1987, $36,3464$.

30. Gerry, C.; Knight, P. Introductory Quantum Optics; Cambridge University Press: Cambridge, UK, 2005.

31. Feldmann, T.; Kosloff, R. Performance of discrete heat engines and heat pumps in finite time. Phys. Rev. E 2000, 61, 4774-4790.

32. Del Campo, A.; Goold, J.; Paternostro, M. More bang for your buck: Super-adiabatic quantum engines. Sci. Rep. 2014, 4, 6208.

33. Plastina, F.; Alecce, A.; Apollaro, T.; Falcone, G.; Francica, G.; Galve, F.; Gullo, N.L.; Zambrini, R. Irreversible work and inner friction in quantum thermodynamic processes. Phys. Rev. Lett. 2014, 113, 260601.

34. Tasaki, H. Jarzynski relations for quantum systems and some applications. arxiv 2000, arxiv:cond-mat/0009244.

35. Evans, D.J.; Cohen, E.; Morriss, G. Probability of second law violations in shearing steady states. Phys. Rev. Lett. 1993, 71, 2401-2404.

36. Crooks, G.E. Entropy production fluctuation theorem and the nonequilibrium work relation for free energy differences. Phys. Rev. E 1999, 60, 2721.

37. Barnett, S.; Radmore, P.M. Methods in Theoretical Quantum Optics; Oxford University Press: Oxford, UK, 2002.

38. Unruh, W.G. Notes on black-hole evaporation. Phys. Rev. D 1976, 14, 870. 
39. Bernard, C.; Duncun, A. Regularization and renormalization of quantum field theory in curved space-time. Ann. Phys. 1977, 107, 201-221.

40. Setare, M.R. Cosmological particle creation and dynamical Casimir effect. Int. J. Theor. Phys. 2004, 43, $2237-2242$.

41. Moradi, S. Creation of Scalar and Dirac Particles in Asymptotically Flat Robertson-Walker Spacetimes. Int. J. Theor. Phys. 2008, 47, 2807-2818.

42. Grib, A.A.; Pavlov, Y.V. Superheavy particles in Friedmann cosmology and the dark matter problem. Int. J. Mod. Phys. D 2002, 11, 433-436.

43. Grib, A.A.; Pavlov, Y.V. Cold dark matter and primordial superheavy particles. Int. J. Mod. Phys. A 2002, 17, 4435-4439.

44. Grib, A.A.; Pavlov, Y.V. Quantum field theory in curved space-time and the early Universe. arXiv 2002, arXiv:gr-qc/0206040.

45. Grib, A.A.; Pavlov, Y.V. On the cosmological coincidence between visible and hidden masses. Grav. Cosmol. Suppl. 2002, 8, 50-51.

46. Vedral, V. Introduction to Quantum Information Science; Oxford University Press: New York, NY, USA, 2006.

47. Kondepudi, D.; Prigogine, I. Modern Thermodynamics; John Wiley and Sons: West Sussex, UK, 2007.

48. Nielsen, M.A.; Chuang, I.L. Quantum Computation and Quantum Information; Cambridge University Press: Cambridge, UK, 2000.

49. Broadbridge, P.; Zulkowski, P. Dark energy states from quantization of boson fields in a universe with unstable modes. Rep. Math. Phys. 2006, 57, 27-40.

(C) 2018 by the authors. Licensee MDPI, Basel, Switzerland. This article is an open access article distributed under the terms and conditions of the Creative Commons Attribution (CC BY) license (http:/ / creativecommons.org/licenses/by/4.0/). 